\title{
THE ROLE OF STACK NON ACOUSTIC PARAMETERS ON THERMODYNAMIC PERFOMANCE OF STANDING WAVE DEVICES
}

\author{
S. Di Filippo*, M. Napolitano, R. Dragonetti, and R. Romano \\ Department of Industrial Engineering, University of Naples Federico II, P.le Tecchio 80, 80125, Naples, Italy. \\ *Corresponding author's e-mail: sabato.difilippo@unina.it
}

Keywords: stack geometry, pore cross-section, non-acoustic parameters

\section{Introduction}

The stack is the core of an thermoacoustic standing wave device. In thermoacoustic engines, heat pumps and refrigerators the geometric characteristics of the stack influence the thermodynamic performance. Until now the stack is studied by using viscous and thermal functions that vary with the shape of the pore. However more advantageous general functions can be taken into account to study a complex cross-section of pores and also the tortuosity. These functions involve classic non-acoustic parameters such as the air-flow resistivity, the tortuosity, the thermal and viscous characteristic length. Aim of this work is to introduce these functions in the classical thermoacoustic theory and to study theoretically the effect on the performance of a thermoacoustic engines.

\section{Thermal and viscous averaged functions}

The use of thermal and viscous averaged functions are introduced in the thermoacoustic theory by Swift [1] to describe the viscous and thermal interaction inside the stack. Similar functions are also used to describe the acoustic properties of a porous material such as the absorption and the reflection coefficient. However a porous material used in practical applications of sound control is quite different from the stack introduced in the Swift theory because do not exist straight but tortuous pores with various interconnections among them. For these reasons, numerous researchers engaged themself to derive more general models to describe the complex geometry inside a porous material. For example, Biot [2] and Stinson [3] later, found that the sound propagation inside a porous material, having straight pores but different cross-section, can be studied with general thermal and viscous averaged functions, by using an equivalent hydraulic radius or a pore-shape factor. In particular Stinson shows that the viscous interaction can be described by the complex density $\tilde{\rho}$ while the thermal interaction can be described by the complex bulk modulus $\widetilde{K}$.

Nowadays, in order to take also into account the tortuosity inside a porous material the most used model is that given by Johnson [4] to describe the viscous interaction and that given by Champoux [5] to describe the thermal interaction. In the Johnson model:

$$
\tilde{\rho}=\frac{\rho_{0} \alpha_{\infty}}{\phi}\left(1+\frac{\sigma \phi}{j \omega \rho_{0} \alpha_{\infty}} \sqrt{1+\frac{4 \alpha_{\infty}^{2} \mu \rho_{0} \omega}{\phi^{2} \sigma^{2} \Lambda_{v}^{2}}}\right)
$$

where $\rho_{0}$ is the air static density, $\mu$ is the dynamic viscosity, $\alpha_{\infty}$ is the high frequency limit of the tortuosity, $\sigma$ is the static air-flow resistivity, $\phi$ is the open porosity and $\Lambda_{v}$ is the viscous characteristic length. In the Champoux model: 


$$
\widetilde{K}=\gamma P_{0}\left(\gamma-\frac{(\gamma-1)}{1+\frac{8 \mu}{j \Lambda_{t}^{2} P_{r} \omega \rho_{0}} \sqrt{1+j \frac{\Lambda_{t}^{2} \rho_{0} \omega}{16 \mu}}}\right)^{-1}
$$

where $\gamma$ is the ratio of isobaric to isochoric specific heats, $P_{0}$ is the static air pressure, $P_{r}$ is the Prandtl number and $\Lambda_{t}$ is the thermal characteristic length. These functions are in turn improved by the Pride [6] and Lafarge [7] but other parameters are introduced. In this preliminary study these further models are not considered.

\section{Use of general thermal and viscous averaged functions in the thermoacoustic theory}

The complex density and the bulk modulus can be related to the function $f_{v}$ and $f_{k}$ used in the Swift theory. A matlab code, tested with DeltaEC, is developed to take into account these new general functions. The non-acoustic parameters can be obtained by numerically solving the thermoacoustic interaction inside a pore without considering the variations in temperature as shown in figure 1.
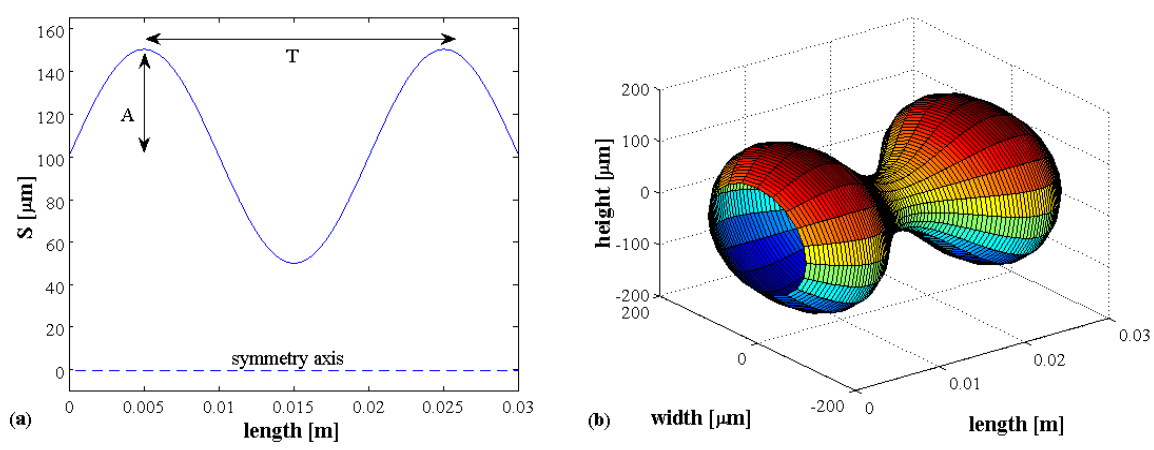

Figure 1: a) Axial symmetric section and b) 3D view of a typical considered pore shape.

It is found that this general approach to study the influence of the stack geometry in terms of porosity, air-flow resistivity, tortuosity and thermal and viscous characteristic length is more advantageous than to use different averaged functions for any pore cross-section.

\section{References}

[1] Backhaus, S., and Swift, G. "A thermoacoustic-Stirling heat engine: detailed study", J. Acoust. Soc. Am., 107, (2000), 3148-3166.

[2] M. A. Biot, "Theory of Propagation of Elastic Waves in a Fluid-Saturated Porous Solid. II. Higher Frequency Range", J. Acoust. Soc. Am., 28 , (1956), 179-191.

[3] Stinson, M. R., The propagation of plane sound waves in narrow and wide circular tubes, and generalization to uniform tubes of arbitrary cross-sectional shape . J. Acoust. Soc. Amer., 89, (1991) 550-8.

[4] Johnson D. L., Koplik J. and Dashen R., Theory of dynamic permeability and tortuosity in fluid-saturated porous media, J. Fluid Mech. 176, (1987), 379-402.

[5] Champoux Y. and Allard J.-F., Dynamic tortuosity and bulk modulus in air-saturated porous media, J. Appl. Phys., 70, (1991), 1975-1979.

[6] Pride S. R., Morgan F. D. and Gangi A. F., Drag forces of porous-medium acoustics, Phys. Rev. B 47, (1993), 4964-4978.

[7] Lafarge D., Lemarinier P., Allard J.-F. and Tarnow V., Dynamic compressibility of air in porous structures at audible frequencies, J. Acoust. Soc. Am. 102, (1997), 1995-2006. 